\title{
Cosmogenic Evidence for Past SEP Events
}

\author{
Fusa Miyake ${ }^{1}$ \\ Institute for Space-Earth Environmental Research, Nagoya University \\ Furo-cho, Chikusa-ku, Nagoya 464-8601, Japan \\ E-mail: fmiyake@isee.nagoya-u.ac.jp
}

\begin{abstract}
Terrestrial cosmogenic nuclides (e.g., ${ }^{14} \mathrm{C},{ }^{10} \mathrm{Be}$, and ${ }^{36} \mathrm{Cl}$ ) are primarily produced by galactic cosmic rays. However, a certain amount of these nuclides is also produced by solar energetic particles (SEPs) derived from sporadic solar events such as solar flares and coronal mass ejections. Cosmic ray increase events in $\mathrm{AD} 774 / 775$, $\mathrm{AD} 993 / 994$ (or AD 992/993), and $\sim \mathrm{BC} 660$ have been discovered using ${ }^{14} \mathrm{C}$ data in tree rings (Miyake et al. 2012, 2013; Park et al. 2017). It is considered that the most plausible cause of these events was an extreme SEP events with very hard energy spectra based on ${ }^{14} \mathrm{C}$ analyses of tree rings and ${ }^{10} \mathrm{Be}$ and ${ }^{36} \mathrm{Cl}$ analyses of ice cores (e.g., Mekhaldi et al. 2015; Miyake et al. 2015, 2019; Büntgen et al. 2018; O'Hare et al. 2019). These SEP events are estimated to be several dozens of times larger than the largest events seen in direct observations, and an event of that size might have a serious impact on modern society. Therefore, it is important to investigate the occurrence rate of past extreme events. In recent years, surveys of past SEP events have been actively conducted via cosmogenic nuclide measurements with high time resolution ( one-year resolution). Here the detected past SEP candidates and a further survey of similar events are reviewed.
\end{abstract}

cosmogenic nuclides

extreme SEP events

36th International Cosmic Ray Conference -ICRC2019-

July 24th - August 1st, 2019

Madison, WI, U.S.A.

${ }^{1}$ Speaker

(C) Copyright owned by the author(s) under the terms of the Creative Commons 


\section{Introduction}

Solar energetic particles (SEPs) are caused by solar explosions, such as solar flares and coronal mass ejections, and are accelerated to high energies up to a few $\mathrm{GeV}$. A phenomenon where the SEP flux increases greatly in the vicinity of the Earth is called an SEP event or a solar proton event (SPE). Direct observations of SEP events have been made by satellites for the past 50 years (e.g., the Geostationary Operational Environmental Satellite). In Addition to direct observations, ground detectors such as neutron monitors have observed SEP events indirectly, i.e., SEP events with hard energy spectra and/or large fluxes which can develop a cascade shower to the ground level (ground level enhancements), have been measured for the past $\sim 70$ years.

A large solar explosion does not necessarily cause an extreme SEP event because the occurrence of SEP events depends on the positions of the solar explosions on the solar surface, e.g., solar explosions that occur on the western side of the Sun, especially around its western limb, generate many SEPs that arrive at the Earth along the interplanetary Parker's magnetic field. Even though a large solar explosion does not necessarily involve SEP events or geomagnetic storms, which can pose serious hazards for a modern society, extreme SEP events tend to occur due to large solar explosions.

While an understanding of extreme SEP events is a very important issue not only to provide an explanation of the mechanism of particle accelerations but also for the fields of space climate and solar physics, observational data from the past $\sim 70$ years alone do not provide information concerning the longer-term behavior of extreme SEP events, e.g., the frequency of extreme SEP events and the upper limit of the size of such events. A clue concerning these problems was recently provided by ${ }^{14} \mathrm{C}$ analyses of annual rings, i.e., it was shown that annual ${ }^{14} \mathrm{C}$ data can be used as proxy data for past SEP events. Since then, annual ${ }^{14} \mathrm{C}$ data collection has been heavily promoted. This paper introduces how ${ }^{14} \mathrm{C}$ and other cosmogenic nuclides $\left({ }^{10} \mathrm{Be}\right.$ and ${ }^{36} \mathrm{Cl}$ in ice cores) have been used in past SEP event studies and the latest results of further SEP event searches using cosmogenic data.

\section{Proxy data of past extreme SEP events}

\subsection{Cosmogenic nuclides as proxy data for past SEP events}

Cosmogenic nuclides such as ${ }^{14} \mathrm{C},{ }^{10} \mathrm{Be}$ and ${ }^{36} \mathrm{Cl}$ are produced by energetic particles, primarily galactic cosmic rays, in the atmosphere. The main channel for the production of ${ }^{14} \mathrm{C}$ is ${ }^{14} \mathrm{~N}(\mathrm{n}, \mathrm{p}){ }^{14} \mathrm{C}$, and those for ${ }^{10} \mathrm{Be}$ and ${ }^{36} \mathrm{Cl}$ are spallation reactions such as ${ }^{14} \mathrm{~N}(\mathrm{n}, \mathrm{x}){ }^{10} \mathrm{Be}$ and ${ }^{40} \mathrm{Ar}(\mathrm{p}, \mathrm{x}){ }^{36} \mathrm{Cl}$ (e.g., Beer et al., 2012). Because typical threshold energies for the production of these nuclides are above $\sim$ tens of $\mathrm{MeV}$, high-energy SEP particles can also produce sufficient amounts of cosmogenic nuclides (e.g., Usoskin, 2017).

Following the formation of cosmogenic nuclides in the atmosphere, ${ }^{14} \mathrm{C}$ is incorporated into tree rings as $\mathrm{CO}_{2}$ in the global carbon cycle; conversely, ${ }^{10} \mathrm{Be}$ and ${ }^{36} \mathrm{Cl}$ are accumulated in ice sheets in polar regions. Therefore, the cosmogenic nuclide concentrations in archival samples such as tree rings and ice cores provide information concerning past cosmic ray intensities. Because SEP events occur over short time periods, with a typical time scale of hours to days, 
extreme SEP events should be recorded as rapid spikes within a single year in cosmogenic nuclide data.

\subsection{Cosmic ray events shown in ${ }^{14} \mathrm{C}$ data}

The tropospheric concentration of ${ }^{14} \mathrm{C}$ is observed to be nearly uniform on a global scale due to the global carbon cycle; therefore, tree-ring ${ }^{14} \mathrm{C}$ data show similar values regardless of location. At the same time, tree-ring ${ }^{14} \mathrm{C}$ data reflect an attenuated and phase-shifted variation of the original cosmic ray variation due to the carbon cycle. This is in contrast to ${ }^{10} \mathrm{Be}$ and ${ }^{36} \mathrm{Cl}$, which can reflect more direct information concerning original cosmic ray variations than ${ }^{14} \mathrm{C}$; however, one needs to determine the cosmic ray variations from multiple ${ }^{10} \mathrm{Be}$ and ${ }^{36} \mathrm{Cl}$ measurements using different archive samples because such measurements are highly sensitive to the deposition process (e.g., climate effects). If we can detect an annual ${ }^{14} \mathrm{C}$ spike, even using only one archive sample, it may be possible to detect extreme SEP events.

Accordingly, Miyake et al. (2012) conducted ${ }^{14} \mathrm{C}$ measurements using a Japanese cedar sample with an annual resolution and detected a sharp increase in the ${ }^{14} \mathrm{C}$ concentration $(\sim 15 \%)$ from $\mathrm{AD} 774$ to $\mathrm{AD} 775$. This increase is significantly larger than the normal ${ }^{14} \mathrm{C}$ annual variation ( $\sim 1 \%$ or less) and the measurement accuracy of ${ }^{14} \mathrm{C}(2-3 \%)$. This event has been then reproduced by ${ }^{14} \mathrm{C}$ analyses using tree samples from around the world (e.g., Büntgen et al. 2018). The ${ }^{14} \mathrm{C}$ increment of the $\mathrm{AD} 774 / 775$ event has been estimated to be 2-3 times larger than the amount of ${ }^{14} \mathrm{C}$ atoms produced by ordinary galactic cosmic rays in a single year (e.g., Büntgen et al. 2018), and several studies have shown that there was a short-term cosmic ray input in the spring-summer of AD 774 (e.g., Büntgen et al. 2018; Uusitalo et al. 2018).

Subsequently, similar rapid ${ }^{14} \mathrm{C}$ increases were reported in AD 993/994 (Miyake et al. 2013), BC 3372/3371 (Wang et al. 2017), and BC 660 (Park et al. 2017).

\subsection{Origin of cosmic ray events}

Not only the SEP events mentioned above but also phenomena emitting gamma rays, such as gamma ray bursts and supernovae, as well as comet impacts on the Earth have been considered as possible causes of rapid ${ }^{14} \mathrm{C}$ increases (e.g., Hambaryan and Neuhäuser 2013; Overholt and Melott 2013). In the case of comet collisions, the diameter of the comet must be very large ( 100 $\mathrm{km}$ ) to cause such a ${ }^{14} \mathrm{C}$ increase and such giant comets would necessarily leave a serious scar on the Earth; however, there are no such craters corresponding to the measured cosmic ray events. Therefore, the comet hypothesis has been abandoned (Overholt and Melott 2013; Usoskin and Kovaltsov 2015). Accordingly, it is necessary to consider the possible gamma ray and SEP (mainly proton) origins. Because it is difficult to pursue this problem using only a single ${ }^{14} \mathrm{C}$ dataset, various validations have been performed.

The first validation is to estimate the type and energy spectrum of the original particles by measuring the concentrations of ${ }^{10} \mathrm{Be}$ and ${ }^{36} \mathrm{Cl}$ in ice cores and determining the production ratios between different nuclides. Measurements of ${ }^{10} \mathrm{Be}$ and ${ }^{36} \mathrm{Cl}$ concentrations in ice cores from Antarctica and Greenland were conducted for the AD 775, AD 993/994, and BC 660 events (Miyake et al. 2015; Sigl et al. 2015; Mekhaldi et al. 2015; Miyake et al. 2019; O'Hare et al. 2019). In all of these events, rapid increases in the ${ }^{10} \mathrm{Be}$ and ${ }^{36} \mathrm{Cl}$ concentrations were detected in both hemispheres. It has been estimated that gamma rays produce an insufficient number of ${ }^{10} \mathrm{Be}$ atoms to be detected, because of the higher threshold energy of ${ }^{10} \mathrm{Be}$ production compared to 
protons (Pavlov et al. 2013); therefore, the existence of clear ${ }^{10} \mathrm{Be}$ peaks are grounds to refute a gamma ray origin (Miyake et al. 2015, 2019; Mekhaldi et al. 2015). In addition, the ${ }^{36} \mathrm{Cl} /{ }^{10} \mathrm{Be}$ ratio indicates that the original particles were consistent with SEPs with very hard energy spectra (Mekhaldi et al. 2015; O'Hare et al. 2019).

The second validation is to investigate the north-south hemispheric symmetry and the latitude dependence of the ${ }^{14} \mathrm{C}$ and ${ }^{10} \mathrm{Be}$ data. In the case of a gamma ray origin, hemispheric symmetry and latitude dependences should not appear because gamma rays are not affected by the geomagnetic field (note that, if gamma rays entered under limited conditions, i.e., incident in the vicinity of the equator, hemispheric symmetry would appear). Carbon-14 data using tree rings in locations worldwide and ${ }^{10} \mathrm{Be}$ data using ice cores from Greenland and Antarctica indicate a similar increment of nuclide concentrations in both hemispheres and a significant latitudinal dependence in the ${ }^{14} \mathrm{C}$ data (Miyake et al. 2015, 2019; Mekhaldi et al. 2015; Büntgen et al. 2018; Uusitalo et al. 2018). These facts also support an SEP origin for these events.

The third validation is to look for historical documents of past auroral records. Because large-scale aurorae (low-latitude aurorae: often observed as red aurorae) occur with large geomagnetic storms, auroral records can provide proxy data for past geomagnetic storms. Recently, Hayakawa et al. examined worldwide auroral records, and reported several records of red aurorae from late AD 992 to early AD 993 in Korea, Germany, and Ireland, which are probably related to the AD 993/994 event (Hayakawa et al. 2017). Even though no historical records were found in AD 774, several records of red aurorae were found in AD 770-773, and AD 776, which indicate higher solar activity in the AD 770s (Hayakawa et al. 2019).

For these reasons, it is thought that the annual cosmic ray events detected in the cosmogenic nuclide data reflect SEP events.

\section{Further surveys of past SEP events and final remarks}

To capture the increases in SEP-derived cosmogenic nuclides, data must be measured with a temporal resolution of one year or less. Therefore, for example, even though 10-year resolution tree-ring ${ }^{14} \mathrm{C}$ data have been acquired over the Holocene (e.g., the ${ }^{14} \mathrm{C}$ age calibration curve: IntCal data) (Reimer et al. 2013), such data cannot reliably capture SEP events. However, if the ${ }^{14} \mathrm{C}$ increase is very large, it should appear in low-resolution IntCal data, e.g., the AD 775 event marks the third-largest increase rate (min-max value) in the IntCal data over the past 10,000 years. Miyake et al. (2017) selected candidate ages of past SEP events comparable to the AD 775 event during the Holocene (15 periods: with the criterion of an increase of more than $0.3 \%$ /year in $\Delta^{14} \mathrm{C}$ ), and 7 periods of ${ }^{14} \mathrm{C}$ concentrations have been measured with an annual resolution so far (Stuiver et al. 1998; Nagaya et al. 2012; Miyake et al. 2017). However, only the AD 775 event shows a rapid (annual) increase; therefore, it is possible that other large ${ }^{14} \mathrm{C}$ increase events that are larger than the AD 775 event have rarely occurred in the past 10,000 years (Miyake et al. 2017).

In recent years, measurements of cosmogenic nuclides with high-time resolution (primarily one-year resolution) have been actively advanced for the exploration of past SEP events (e.g., Jull et al. 2018; Eastoe et al. 2019). For example, many researchers have measured ${ }^{14} \mathrm{C}$ concentrations with one-year resolution using different tree samples and these data will be adopted 
in the next IntCal dataset. Such collections of annual data continue, and therefore it is expected that more long-term data will be obtained in the future. Because past SEP event surveys require the use of multiple archive samples and nuclides, it is important for researchers to collaborate and collect more data. Such data will provide more accurate information concerning the occurrence rate and the upper limit of extreme SEP events.

\section{Acknowledgements}

This manuscript was supported by the Japan Society for the Promotion of Science (JSPS) Grantin-Aid for Scientific Research (16H06005).

\section{References}

[1] Beer, J., McCracken, K., and von Steiger, R., Springer, Berlin (2012).

[2] Büntgen, U., et al., Nat. Commun., 9:3605, DOI: 10.1038/s41467-018-06036-0 (2018).

[3] Eastoe, C.J., Tucek, C.S. and Touchan, R., Radiocarbon, 61(3), 661-680 (2019).

[4] Hambaryan, V. V. and Neuhäuser, R., MNRAS, 430, 32-36 (2013).

[5] Hayakawa, H., et al., Solar Phys. 292, 12 (2017).

[6] Hayakawa, H., et al., Solar Phys. 294, 42 (2019).

[7] Jull, A.J.T., et al., Radiocarbon 60, 1237-1248 (2018).

[8] Mekhaldi, F., et al., Nat. Commun. 6:8611, DOI: 10.1038/ncomms9611 (2015).

[9] Miyake, F., Nagaya, K., Masuda, K., and Nakamura, T., Nature, 486, 240-242 (2012).

[10] Miyake, F., Masuda, K. and Nakamura, T., Nat. Commun., 4, 1748, DOI: 10.1038/ncomms2873 (2013).

[11] Miyake, F., et al., Geophys. Res. Lett., 42, 84-89 (2015).

[12] Miyake, F., et al., Geophys. Res. Lett., 46, 11-18 (2019).

[13] O’Hare, P., et al., PNAS, 116(13), 5961-5966 (2019).

[14] Overholt, A. C. and Melott, A. L., EPSL, 377-378, 55-61 (2013).

[15] Park, J., et al., Radiocarbon, 59(4), 1147-1156 (2017).

[16] Pavlov, A. K., et al., MNRAS, 435(4), 2878-2884 (2013).

[17] Reimer, P.J., et al., Radiocarbon 55, 1869-1887 (2013).

[18] Sigl, M., et al., Nature 523, 543-549 (2015).

[19] Usoskin, I. G. and Kovaltsov, G. A., Icarus, 260, 475-476 (2015).

[20] Usoskin, I. G., Living Reviews in Solar Physics, 14:3 (2017). 